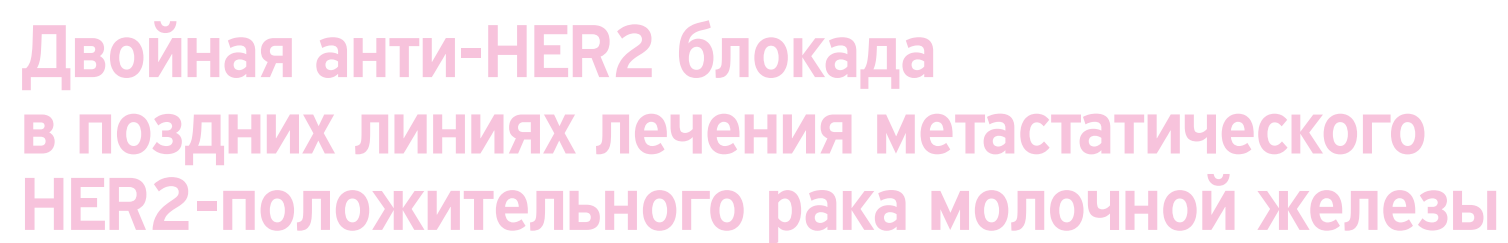

М.А.Фролова ${ }^{\bowtie}$, https://orcid.org/0000-0002-8149-0085, drfrolova@yandex.ru

М.Б. Стенина, mstenina@mail.ru

Национальный медицинский исследовательский центр онкологии имени Н.Н. Блохина; 115478, Россия, Москва, Каширское шоссе, д. 24

\title{
Резюме
}

В последние годы появились широкие возможности лечения больных метастатическим HER2-положительным раком молочной железы, благодаря чему продолжительность жизни этой категории больных сегодня является наибольшей по сравнению с другими подтипами. Добавление пертузумаба к трастузумабу и доцетакселу продемонстрировало увеличение общей выживаемости, в связи с чем данный режим признан стандартом 1-й линии лечения. Оптимальным вариантом 2-й линии терапии является трастузумаб эмтанзин. Далее могут применяться различные комбинации цитостатиков и анти-HER2 направленных агентов. Представляется интересным выбор тактики в поздних линиях лечения тех больных, которые ранее не получали пертузумаб: стоит ли его назначать и с чем лучше комбинировать. Одним из возможных вариантов является комбинация эрибулина с двойной анти-HER2 блокадой трастузумабом и пертузумабом. Эрибулин является антимикротрубочковым агентом, вызывает необратимую блокаду митоза. Кроме того, он обладает немитотическими эффектами: в экспериментах in vitro и in vivo продемонстрирована его способность восстанавливать нормальную васкуляризацию опухоли, уменьшать степень гипоксии и, как следствие, снижать способность опухолевых клеток к миграции и инвазии. В данной статье представлен клинический случай применения эрибулина с двойной анти-HER2 блокадой в 6-й линии лечения больной метастатическим НЕR2положительным раком молочной железы. Был продемонстрирован длительный контроль болезни (в течение двух лет) с удовлетворительным качеством жизни.

Ключевые слова: HER2-положительный метастатический рак молочной железы, двойная анти-HER2 блокада, трастузумаб, пертузумаб, эрибулин

Для цитирования: Фролова М.А., Стенина М.Б. Двойная анти-HER2 блокада в поздних линиях лечения метастатического HER2-положительного рака молочной железы. Медицинский совет. 2021;(20):156-159. https://doi.org/10.21518/2079701X-2021-20-156-159.

Конфликт интересов: авторы заявляют об отсутствии конфликта интересов.

\section{Dual anti-HER2 blockade in late-line treatment of metastatic HER2-positive breast cancer}

Mona A. Frolova ${ }^{\varpi}$, https://orcid.org/0000-0002-8149-0085, drfrolova@yandex.ru

Marina B. Stenina, mstenina@mail.ru

Blokhin National Medical Research Center of Oncology; 24, Kashirskoe Shosse, Moscow, 115478, Russia

\begin{abstract}
In recent years, there has been a wide range of treatment options for patients with metastatic HER2-positive breast cancer, resulting in the highest life expectancy for these patients among all subtypes. The addition of pertuzumab to trastuzumab and docetaxel has been shown to increase overall survival and is therefore recognized as the standard first-line treatment. The most optimal second-line treatment option is trastuzumab emtansine. In addition, various combinations of cytostatics and anti HER2 targeting agents can be used. The choice of treatment options in heavily pretreated patients is of great interest. If they have not previously received pertuzumab, is it worth to use it and which combination is the best? One possible option is the combination of eribulin with the dual anti-HER2 blockade with trastuzumab and pertuzumab. Eribulin is an anti-microtubule agent that irreversibly blocks mitosis. In addition, it has non-mitotic effects - in vivo and in vitro experiments demonstrated its ability to restore normal tumor vascularization, reduce the area of hypoxia and, as a consequence, decrease tumor cells migration and invasion. This article represents a clinical case of the use of eribulin with double anti-HER2 blockade in the 6th line of treatment in a patient with metastatic HER2-positive breast cancer. Long-term control of the disease (within 2 years) with a satisfactory quality of life has been demonstrated.
\end{abstract}

Keywords: HER2-positive metastatic breast cancer, double anti-HER2 blockade, trastuzumab, pertuzumab, eribulin

For citation: Frolova M.A., Stenina M.B. Dual anti-HER2 blockade in late-line treatment of metastatic HER2-positive breast cancer. Meditsinskiy sovet = Medical Council. 2021;(20):156-159. (In Russ.) https://doi.org/10.21518/2079-701X-2021-20-156-159.

Conflict of interest: the authors declare no conflict of interest. 


\section{ВВЕДЕНИЕ}

Внедрение в клиническую практику анти-HER2 (Human Epidermal Growth Factor Receptor 2) направленной терапии и в частности трастузумаба позволило кардинально изменить судьбу больных ранним и распространенным HER2положительным раком молочной железы (РМЖ). На сегодняшний день медиана продолжительности жизни больных метастатическим HER2-положительным РМЖ, особенно с экспрессией гормональных рецепторов, является наибольшей по сравнению с другими подтипами $[1,2]$. Это стало возможным благодаря использованию новых препаратов анти-HER2 направленного действия с различным механизмом действия, таких как лапатиниб, пертузумаб, T-DM1. В 2011 г. стали известны результаты исследования CLEOPATRA [3], которое показало, что добавление пертузумаба к комбинации трастузумаба и доцетаксела в 1-й линии лечения метастатического HER2-позитивного РМЖ привело К увеличению медианы времени до прогрессирования с 12,4 до 18,5 мес. (относительный риск (ОР) 0,65; 95\% доверительный интервал (ДИ) 0,54-0,78; р < 0,001). На основании этого в июне 2012 г. FDA (Food and Drug Administration Управление по санитарному надзору за качеством пищевых продуктов и медикаментов (ША) утвердило комбинацию пертузумаба, трастузумаба и доцетаксела в качестве стандарта 1-й линии лечения метастатического HER2позитивного РМЖ ${ }^{1}$. Позднее были показаны результаты по общей выживаемости, и оказалось, что добавление пертузумаба приводит к значимому, на 15,7 мес., увеличению медианы продолжительности жизни (с 40,8 до 56,5 мес.) [4].

Еще одним препаратом с принципиально новым механизмом действия, воздействующим на HER2-позитивный PMЖ, стал T-DM1 (трастузумаб эмтанзин), представляющий собой конъюгат трастузумаба с цитотоксическим агентом, стабилизирующим микротрубочки. Такое сочетание позволяет противоопухолевым агентам проникать непосредственно в HER2-позитивные опухолевые клетки, минимизируя воздействие на здоровые ткани. В 2012 г. стали известны результаты рандомизированного исследования III фазы EMILIA, в котором T-DM1 применялся уже у пациентов, ранее получавших лечение трастузумабом и таксанами. В качестве группы сравнения использовалась стандартная на тот момент комбинация капецитабина + лапатиниба [5]. Частота объективных эффектов была выше в группе T-DM1 (43,6\%; $95 \%$ ДИ 38,6-48,6) против группы сравнения (30,8\%; 95\% ДИ 26,335,7; p < 0,001). Лечение в группе Т-DM1 привело к значительному улучшению отдаленных результатов: медиана времени до прогрессирования при лечении T-DM1 составила 9,6 и 6,4 мес. - в группе сравнения (ОР 0,65; 95\% ДИ 0,550,77; р 0,001). Увеличение выживаемости в группе T-DM1 наблюдалось независимо от линии терапии по поводу метастатического процесса, а также у больных при прогрессировании после адъювантной или неоадъювантной терапии раннего рака. При промежуточном анализе общей выживаемости T-DM1 значительно увеличивал медиану продолжительности жизни по сравнению с группой «капецита-

\footnotetext{
${ }_{1}^{1}$ PERJETA ${ }^{\circledast}$ (pertuzumab). Available at: https://www.accessdata.fda.gov/drugsatfda_docs/
} label/2013/125409s051lbl.pdf. бин + лапатиниб» (30,9 и 25,1 мес.; ОР 0,68; 95\% ДИ 0,550,85; $p$ < 0,001). Частота нежелательных явлений 3-4-й степени была выше при использовании комбинации «капецитабин + лапатиниб», чем при лечении T-DM1 (57 и 40,8\%). На основании более высокой эффективности и относительно низкой токсичности T-DM1 был признан стандартом 2-й линии терапии метастатического HER2-позитивного РМЖ.

Таким образом, на сегодняшний день сложился алгоритм лечения диссеминированного HER2-положительного рака молочной железы: 1-я линия - таксаны в комбинации с трастузумабом и пертузумабом; 2-я линия - T-DM1, далее лапатиниб + капецитабин и различные цитостатики в комбинации с трастузумабом [6]. Однако в реальной клинической практике данный алгоритм не всегда выполним в силу ряда причин. Возникает вопрос: если пертузумаб не использовался в 1-й линии лечения, можем ли мы применять этот препарат в последующем и с какими цитостатиками. Одним из последних цитостатиков, вошедших в клиническую практику для лечения больных РМЖ, является эрибулин.

Эрибулин представляет собой синтетический аналог халихондрина В - соединения, которое исходно было выделено из морской губки. Так же как и таксаны и винкаалкалоиды, эрибулин является антимикротрубочковым агентом. По механизму действия эрибулин, в отличие от таксанов, подавляет полимеризацию, но не влияет на процесс деполимеризации микротрубочек. Соединяясь с $\beta$-тубулином на положительном конце микротрубочки, эрибулин приводит к ингибированию роста микротрубочек и секвестрации их в неактивные конгломераты [7, 8]. В результате этого останавливается деление клетки во время митоза и клетка вступает в апоптоз $[9,10]$. В отличие от других микротрубочковых агентов, эрибулин приводит к необратимому блокированию митоза, а также активен при мутациях $\beta$-тубулина $[11,12]$. Помимо воздействия на микротрубочки, эрибулин обладает рядом других немитотических эффектов в отношении опухолевых клеток. Так, под воздействием эрибулина происходит восстановление нормальной васкуляризации опухоли, в результате чего уменьшается зона гипоксии внутри опухоли [13].Улучшение перфузии и уменьшение гипоксии, в свою очередь, приводят к мезенхимально-эпителиальному переходу $[14,15]$, который, в отличие от обратного процесса, способствует снижению миграции и инвазии опухолевых клеток [16]. В исследованиях in vitro и in vivo было показано, что под воздействием эрибулина повышается экспрессия эпителиальных и снижается экспрессия мезенхимальных маркеров [17]. В итоге уменьшается агрессивность опухоли и, возможно, повышается чувствительность к последующим линиям химиотерапии [18]. В клинических исследованиях EMBRACE [19] и 301 [20] изучалась эффективность эрибулина в сравнении с терапией по выбору врача или капецитабином у больных, получавших ранее химиотерапию по поводу метастатической болезни. В объединенном анализе, включавшем 1644 больных, было показано увеличение общей продолжительности жизни с 12,6 до 15,0 мес. в группе эрибулина (ОР 0,85, 95\% ДИ 0,768-0,948, p = 0,002) [21]. В исследования включались больные со всеми подтипами РМЖ, включая HER2-положительный, однако анти-HER2 направленная терапия в то время не назначалась. По этой причине 
в финальный анализ больные с HER2-положительными опухолями не вошли. По результатам этих исследований эрибулин был зарегистрирован для применения у больных HER2-отрицательным РМЖ после прогрессирования на терапии антрациклинами и таксанами. В дальнейшем стали проводиться исследования и у больных HER2-положительным раком в сочетании с анти-HER2 терапией. В исследовании II фазы изучалась эффективность комбинации эрибулина и трастузумаба в 1-й линии лечения метастатического HER2положительного РМЖ [22]. Частота объективного ответа составила 71,2\%, а медиана времени без прогрессирования - 11,6 мес., что было сопоставимо с эффективностью доцетаксела и трастузумаба в исследовании CLEOPATRA.

В настоящей статье мы приводим результаты собственного клинического наблюдения применения эрибулина с двойной анти-HER2 блокадой в поздних линиях лечения пациентки с метастатическим HER2-положительным РMЖ.

\section{КЛИНИЧЕСКИЙ СЛУЧАЙ}

Больная Т., 38 лет. В феврале 2008 г. диагностирован рак правой молочной железы сT4N3M0. Дважды была выполнена core-биопсия опухоли. Гистологически - комплексы внутрипротокового, безинвазивного рака. Однако с учетом клинической картины (отек и гиперемия кожи, инфильтрация ткани молочной железы), а также цитологически верифицированного поражения аксиллярных лимфоузлов было решено начать лечение с неоадъювантной химиотерапии. С 21 февраля по 26 июня 2008 г. были проведены 6 курсов индукционной химиотерапии по схеме FAC (доксорубицин $50 \mathrm{Mг} / \mathrm{M}^{2}+$ циклофосфамид $500 \mathrm{мг} / \mathrm{M}^{2}+5$-фторурацил 500 мг/м²) внутривенно капельно 1 раз в 3 нед. с некоторым положительным эффектом. 29 июля 2008 г. выполнена радикальная мастэктомия справа. Гистологическое заключение: инвазивный протоковый рак с метастазами в 8 лимфоузлах. рецепторы к эстрогенам (РЭ) +, рецепторы к прогестерону (РП) +, HER2 3+. С 18 августа по 10 декабря 2008 г. проведены 6 курсов адъювантной химиотерапии по схеме «доцетаксел + циклофосфамид». Проведена адъювантная лучевая терапия на послеоперационный рубец и регионарные зоны в суммарной очаговой дозе (СОД) 44 Гр. С декабря 2008 по декабрь 2009 г. пациентка получала адъювантную терапию трастузумабом 6 мг/кг 1 раз в 3 нед. и тамоксифеном 20 мг/сут внутрь ежедневно. В ноябре 2009 г. появилась гиперемия в области послеоперационного рубца. Антигистаминная и противовоспалительная терапии - без эффекта. В январе 2010 г. выявлена опухолевая инфильтрация в области послеоперационного рубца (при гистологическом исследовании - комплексы раковых клеток). Проведены 6 курсов химиотерапии по схеме «паклитаксел + трастузумаб + эверолимус» с полной регрессией опухолевого процесса. До декабря 2010 г. получала поддерживающую терапию трастузумабом в монорежиме. В январе 2011 г. Вновь отмечено появление опухолевой инфильтрации в зоне послеоперационного рубца. С 8 февраля 2011 по 20 марта 2012 г. проведены 17 курсов химиотерапии по схеме «капецитабин + лапатиниб» с полным эффектом после трех курсов. При контрольном обследовании в марте
2012 г. выявлено прогрессирование за счет увеличения площади опухолевой инфильтрации в области послеоперационного рубца. С 13 апреля по 28 августа 2012 г. проведены 6 курсов химиотерапии по схеме «винорелбин + трастузумаб». В июле 2012 г. выявлено вторичное поражение левой молочной железы. Выполнена биопсия опухоли. Гистологически - инфильтративный дольковый рак 2-й ст. злокачественности. РЭ 0 баллов, РП 0 баллов, HER2 3+, Кі-67 75\%. С 13 августа по 5 сентября 2012 г. проведен курс лучевой терапии на область левой молочной железы в СОД 50 изоГр, левых аксиллярных лимфоузлов в СОД 46 изоГр. С сентября 2012 года по май 2013 г. получала поддерживающую терапию по схеме «лапатиниб + трастузумаб», на фоне которой отмечено дальнейшее прогрессирование опухолевого процесса в области передней грудной стенки справа. С июня 2013 по сентябрь 2014 г. получала терапию T-DM1 с полным эффектом после 2-го курса. В сентябре 2014 г. вновь появились внутрикожные образования в зоне послеоперационного рубца, а также неврологические симптомы (головокружение, головные боли). При МРТ выявлено очаговое образование в правой гемисфере мозжечка размерами 3,4 × 2,8 см. 10 ноября 2014 г. выполнено удаление этого образования, далее проводилась лучевая терапия на послеоперационную полость в СОД 30 Гр. С 17 декабря 2014 по октябрь 2015 г. получала метрономную химиотерапию по следующей схеме: эндоксан 50 мг/сут внутрь + метотрексат по 2,5 мг 2 раза в день 2 раза в неделю внутрь + лапатиниб 1250 мг/сут внутрь ежедневно со стабилизацией опухолевого процесса. В связи с дальнейшим прогрессированием опухолевого процесса с ноября 2015 г. начата терапия по схеме «эрибулин 1,4 мг/м² в 1-й и 8-й дни + трастузумаб 6 мг/кг + пертузумаб 840 мг (нагрузочная доза), затем 420 мг каждые 3 нед.», на фоне которой была достигнута стабилизация опухолевого процесса. Переносимость терапии была удовлетворительной, из побочных эффектов следует отметить нейтропению 2-3-й ст., диарею 1-2-й ст. Терапия продолжалась до октября 2017 г. Далее в связи с прогрессированием опухолевого процесса (появления метастазов в легких) переведена на терапию паклитакселом и трастузумабом. В мае 2018 г. пациентка погибла от прогрессирования опухолевого процесса. Таким образом, общая продолжительность жизни после появления метастатической болезни составила более 8 лет.

\section{ОБСУЖДЕНИЕ}

Стандартом 1-й линии лечения при метастатическом HER2-положительном РМЖ является комбинация таксанов с двойной анти-HER2 блокадой трастузумабом и пертузумабом. Однако в реальной клинической практике это не всегда возможно. Часто у клиницистов возникает вопрос, можем ли мы использовать пертузумаб в поздних линиях лечения, если он не применялся ранее. И если да, то с каким цитостатиком его лучше комбинировать. Клинических исследований, посвященных данному вопросу, немного. Одним из них является исследование II фазы, в котором изучалась эффективность комбинации эрибулина с трастузумабом и пертузумабом у предлечен- 
ных пациенток [23]. Медиана предшествующих линий химиотерапии по поводу метастатической болезни составила 3,5 (1-9). Фармакокинетика эрибулина в этой комбинации не отличалась от фармакокинетики препарата в монорежиме. Частота объективного ответа составила 34,8\%, медиана времени без прогрессирования 42,6 нед. Наиболее частым побочным явлением была нейтропения 3-4-й степени (66,7\% больных).

В приведенном нами клиническом случае пациентка получала комбинацию эрибулина с трастузумабом и пертузумабом в качестве 6-й линии лечения по поводу метастатического HER2-положительного РМЖ. В результате данной терапии была достигнута длительная стабилизация болезни (в течение двух лет!) с удовлетворительным качеством жизни. В предшествующих линиях терапии пациентка полу- чала различные комбинации цитостатиков с трастузумабом, лапатинибом, T-DM1. Важно отметить, что пациентка ранее получила лечение по поводу метастатического поражения головного мозга, после которого прожила 3 года и 9 мес.

\section{ЗАКЛЮЧЕНИЕ}

Данный клинический случай демонстрирует возможность длительного контроля болезни на фоне терапии эрибулином с двойной анти-HER2 блокадой трастузумабом и пертузумабом в поздних линиях лечения метастатического HER2-положительного РМЖ.

Поступила / Received 27.09.2021

Поступила после рецензирования / Revised 15.10.2021 Принята в печать / Accepted 23.10.2021

\section{Список литературы / References}

1. Dawood S., Broglio K., Buzdar A.U., Hortobagyi G.N., Giordano S.H. Prognosis of women with metastatic breast cancer by HER2 status and trastuzumab treatment: an institutional-based review. J Clin Oncol. 2010;28(1):92-98. https://doi. org/10.1200/jco.2008.19.9844

2. Lobbezoo DJ., van Kampen RJ., Voogd A.C., Dercksen M.W., van den Berkmortel F., Smilde TJ. et al. Prognosis of metastatic breast cancer subtypes: the hormone recep tor/HER2-positive subtype is associated with the most favorable outcome. Breast Cancer Res Treat. 2013;141(3):507-514. https://doi.org/10.1007/s10549-013-2711-y.

3. Baselga J., Cortés J., Kim S.B., Im S.A., Hegg R., Im Y.H. et al. Pertuzumab plus trastuzumab plus docetaxel for metastatic breast cancer. N Engl J Med. 2012;366(2):109-119. https://doi.org/10.1056/NEJMoa1113216.

4. Swain S.M., Baselga J., Kim S.B., Ro J., Semiglazov V., Campone M. et al. Pertuzumab, trastuzumab, and docetaxel in HER2-positive metastatic breast cancer. N Engl J Med. 2015;372(8):724-734. https://doi.org/10.1056/NEJMoa1413513.

5. Verma S., Miles D., Gianni L., Krop I.E., Welslau M., Baselga J. et al. Trastuzumab emtansine for HER2-positive advanced breast cancer. N Engl J Med. 2012;367(19):1783-1791. https://doi.org/10.1056/NEJMoa1209124.

6. Cardoso F., Paluch-Shimon S., Senkus E., Curigliano G., Aapro M.S., André F. et al. $5^{\text {th }}$ ESO-ESMO international consensus guidelines for advanced breast cancer (ABC 5). Ann Oncol. 2020;31(12):1623-1649. https://doi.org/10.1016/j. annonc.2020.09.010.

7. Doodhi H., Prota A.E., Rodríguez-García R., Xiao H., Custar D.W., Bargsten K. et al. Termination of Protofilament Elongation by Eribulin Induces Lattice Defects that Promote Microtubule Catastrophes. Curr Biol. 2016;26(13):1713-1721. https://doi. org/10.1016/j.cub.2016.04.053.

8. Smith J.A., Wilson L., Azarenko O., Zhu X., Lewis B.M., Littlefield B.A., Jordan M.A. Eribulin binds at microtubule ends to a single site on tubulin to suppress dynamic instability. Biochemistry. 2010;49(6):1331-1337. https://doi.org/10.1021/bi901810u.

9. Okouneva T., Azarenko O., Wilson L., Littlefield B.A., Jordan M.A. Inhibition of centromere dynamics by eribulin (E7389) during mitotic metaphase. Mol Cancer Ther. 2008:7(7):2003-2011. https://doi.org/10.1158/1535-7163.MCT-08-0095.

10. Mc Gee M.M. Targeting the Mitotic Catastrophe Signaling Pathway in Cancer. Mediators Inflamm. 2015;146282. https://doi.org/10.1155/2015/146282.

11. Towle MJ., Salvato K.A., Wels B.F., Aalfs K.K., Zheng W., Seletsky B.M. et al. Eribulin induces irreversible mitotic blockade: implications of cell-based pharmacodynamics for in vivo efficacy under intermittent dosing conditions. Cancer Res. 2011;71(2):496-505. https://doi.org/10.1158/0008-5472.CAN-10-1874.

12. Wilson L., Lopus M., Miller H.P., Azarenko O., Riffle S., Smith J.A., Jordan M.A. Effects of eribulin on microtubule binding and dynamic instability are strengthened in the absence of the $\beta$ III tubulin isotype. Biochemistry. 2015;54(42):64826489. https://doi.org/10.1021/acs.biochem.5b00745.

13. Funahashi Y., Okamoto K., Adachi Y., Semba T., Uesugi M., Ozawa Y. et al. Eribulin mesylate reduces tumor microenvironment abnormality by vascular remodeling in preclinical human breast cancer models. Cancer Sci. 2014;105(10):1334-1342. https://doi.org/10.1111/cas.12488.

14. Jung H.Y., Fattet L., Yang J. Molecular pathways: linking tumor microenvironment to epithelial-mesenchymal transition in metastasis. Clin Cancer Res. 2015;21(5):962-968. https://doi.org/10.1158/1078-0432.CCR-13-3173.

15. Kashiwagi S., Asano Y., Goto W., Takada K., Takahashi K., Hatano T. et al. Mesenchymal-epithelial Transition and Tumor Vascular Remodeling in Eribulin Chemotherapy for Breast Cancer. Anticancer Res. 2018;38(1):401-410. https://doi.org/10.21873/anticanres.12236.

16. Yoshida T., Ozawa Y., Kimura T., Sato Y., Kuznetsov G., Xu S. et al. Eribulin mesilate suppresses experimental metastasis of breast cancer cells by reversing phenotype from epithelial-mesenchymal transition (EMT) to mesenchymal-epithelial transition (MET) states. Br J Cancer. 2014;110(6):1497-1505. https://doi. org/10.1038/bjc.2014.80.

17. Dybdal-Hargreaves N.F., Risinger A.L., Mooberry S.L. Regulation of E-cadherin localization by microtubule targeting agents: rapid promotion of cortical E-cadherin through p130Cas/Src inhibition by eribulin. Oncotarget. 2017;9(5):5545-5561. https://doi.org/10.18632/oncotarget.23798.

18. Ozawa Y., Okamoto K., Adachi Y., Asano M., Tabata K., Funahashi Y., Matsui J. 36 Suppression of metastasis and improvement of drug distribution by eribulin mesylate. EJC. 2014;50(6 Suppl.):17. https://doi.org/10.1016/S09598049(14)70162-5.

19. Cortes J., O'Shaughnessy J., Loesch D., Blum J.L., Vahdat L.T., Petrakova K. et al. Eribulin monotherapy versus treatment of physician's choice in patients with metastatic breast cancer (EMBRACE): a phase 3 open-label randomised study. Lancet. 2011;377(9769):914-923. https://doi.org/10.1016/S0140-6736(11)60070-6.

20. Kaufman P.A., Awada A., Twelves C., Yelle L., Perez E.A., Velikova G. et al. Phase III open-label randomized study of eribulin mesylate versus capecitabine in patients with locally advanced or metastatic breast cancer previously treated with an anthracycline and a taxane. J Clin Oncol. 2015;33(6):594-601. https://doi. org/10.1200/JCO.2013.52.4892.

21. Twelves C., Cortes J., Vahdat L., Olivo M., He Y., Kaufman P.A., Awada A. Efficacy of eribulin in women with metastatic breast cancer: a pooled analysis of two phase 3 studies. Breast Cancer Res Treat. 2014;148(3):553-561. https://doi. org/10.1007/s10549-014-3144-y.

22. Wilks S., Puhalla S., O'Shaughnessy J., Schwartzberg L., Berrak E., Song J. et al. Phase 2, multicenter, single-arm study of eribulin mesylate with trastuzumab as first-line therapy for locally recurrent or metastatic HER2-positive breast cancer. Clin Breast Cancer 2014:14(6):405-412. https://doi.org/10.1016/j.clbc.2014.04.004

23. Araki K., Fukada I., Yanagi H., Kobayashi K., Shibayama T., Horii R. et al. First report of eribulin in combination with pertuzumab and trastuzumab for advanced HER2-positive breast cancer. Breast. 2017;35:78-84. https://doi.org/10.1016/j. breast.2017.06.015.

\section{Информация об авторах:}

Фролова Мона Александровна, д.м.н., старший научный сотрудник онкологического отделения лекарственных методов лечения (химиотерапевтического) №1, Национальный медицинский исследовательский центр онкологии имени Н.Н. Блохина; 115478, Россия, Москва, Каширское шоссе, д. 24; drfrolova@yandex.ru

Стенина Марина Борисовна, д.м.н., ведущий научный сотрудник онкологического отделения лекарственных методов лечения (химиотерапевтического) №1, Национальный медицинский исследовательский центр онкологии имени Н.Н. Блохина; 115478, Россия, Москва, Каширское шоссе, д. 24; mstenina@mail.ru

Information about the authors:

Mona A. Frolova, Dr. Sci. (Med.), Senior Researcher, Oncology Department of Medicinal Methods of Treatment (Chemotherapy) No. 1, Blokhin National Medical Research Center of Oncology; 24, Kashirskoe Shosse, Moscow, 115478, Russia; drfrolova@yandex.ru

Marina B. Stenina, Dr. Sci. (Med.), Leading Researcher, Oncology Department of Medicinal Methods of Treatment (Chemotherapy) No. 1, Blokhin National Medical Research Center of Oncology; 24, Kashirskoe Shosse, Moscow, 115478, Russia; mstenina@mail.ru 